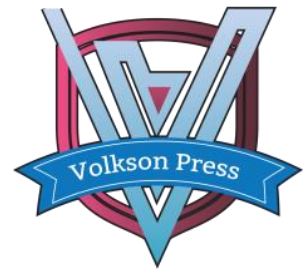

Contents List available at VOLKSON PRESS

Economics \& Management Innovations(EMI)

DOI : http://doi.org/10.26480/icemi.01.2017.320.324

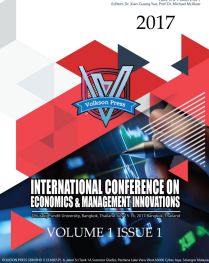

\title{
Effect of Machine Failure Prediction on Selected Parameters of Manufacturing Schedule in a Job-shop Environment
}

Łukasz Sobaszek ${ }^{1, *}$, Arkadiusz Gola ${ }^{1}$ and Edward Kozłowski ${ }^{2}$

${ }^{1}$ Lublin University of Technology, Institute of Technological Systems of Information, Nadbystrzycka 36, 20-618 Lublin, Poland ${ }^{2}$ Lublin University of Technology, Department of Quantitative Methods in Management, Nadbystrzycka 36, 20-618 Lublin, Poland

*l.sobaszek@pollub.pl

This is an open access article distributed under the Creative Commons Attribution License, which permits unrestricted use, distribution, and reproduction in any medium, provided the original work is properly cited.

\section{ARTICLE DETAILS}

\section{Article History:}

Received 02 october 2017 Accepted 06 october 2017 Available online 11 october 2017

\section{Keywords:}

machine failure, prediction, robust scheduling, job shop scheduling, management.

\section{ABSTRACT}

Production scheduling involves a number of factors, which may disrupt or completely block the smooth operation of a production processes. This paper seeks to address one of the key factors involved, machine failure, which is capable of introducing disorganisation and nervousness into production. The relevance of machine failure to production is highlighted by the extensive research in the field of robust scheduling, as a method to absorb potential disruptions. The aim of this paper is to present technological machine failure prediction method to provide the base for a robust schedule, which is subsequently verified by the mathematical model. Furthermore, the paper elaborates on the issue of scheduling in job shop environment and the idea of robust scheduling.

\section{Introduction}

Job scheduling in typical manufacturing systems is the subject of numerous analyses and research works. The research is predominantly focused on scheduling problems in job-shop environments due to the fact that this is the most popular and widely applied method of organising work in production companies [1]. A problem with much of the literature regarding job scheduling is that the work is frequently based on a set of simplifications, which determine that the character of such analyses is largely theoretical [2]. Consequently, a number of problems existing in an actual scheduling of manufacturing processes are disregarded. Practice shows that each production process involves a series of factors that are counter-productive to the realisation of the process itself [3]. One problem that should not be underestimated, as it shows great potential for disrupting production, is machine failure [4].

This paper takes a new look on robust scheduling of manufacturing jobs. The scope of the analysis included technological machine failure prediction and estimation of service times. The data for simulation was obtained from historical records from the Maintenance Department of a production company.

\section{Job-shop production systems}

\subsection{Job shop environment characteristics}

In order to model a job-shop scheduling problem the following sets must be defined $[5,6]$ :

- $\quad M$, which is a set of $m$ machines processing the jobs:

$M=\left\{M_{1}, M_{2}, \ldots, M_{m}\right\}$

$J$, which is a set of $n$ jobs to be processed:

$J=\left\{J_{1}, J_{2}, \ldots, J_{n}\right\}$.

Processing job $J_{i}$ on machine $M_{j}$ will be referred to as operation. Therefore what must be defined is $[5,6]$ :

matrix of machine orders $M O$ of the size $m \times n$ representing the rank of jobs on particular machines (machine routes):

$M O=M_{2}\left[\begin{array}{cccc}J_{1} & J_{2} & \ldots & J_{n} \\ \ldots \\ M_{m}\end{array}\left[\begin{array}{cccc}o_{11} & o_{21} & \ldots & o_{n 1} \\ o_{12} & o_{22} & \ldots & o_{n 2} \\ \ldots & \ldots & o_{i j} & \ldots \\ o_{1 m} & o_{2 m} & \ldots & o_{n m}\end{array}\right]\right.$,

$M O=\left[o_{i j}\right]$

where: $o_{i j}$ - a number describing the rank of operations $i$ on machine $j$ taking the following values:

$$
o_{i j}= \begin{cases}0, & \text { when operation } i \text { is not processed on machine } \\ \{1, \ldots, m\}, & j\end{cases}
$$

where: $m$ - the number of machines processing jobs.

- The matrix of processing times PT:

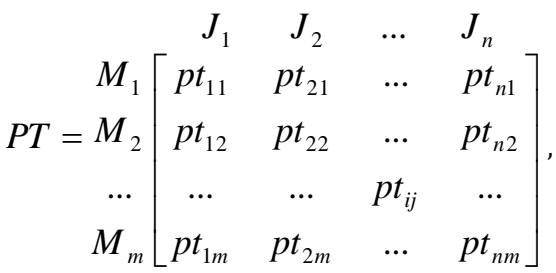


where: $p_{i j}$ - the number describing the processing time of job $i$ on machine j; where:

$$
\bigwedge_{o_{i j}=0} p t_{i j}=0
$$

The rudimentary problem of job scheduling consists in proper assignment of jobs from set $J$ among given machines of set $M$. Scheduling in a job shop environment assumes full scheduling of jobs under technological constraints [7].

It is moreover of certain importance that the developed schedule should be optimal regarding the objective function [6]. There are several objective functions, however, in practice the one that is most widely applied (both in analysis of test problems and scheduling in industrial environment) is "minimise the makespan" $\left(C_{\max }\right)[8,9]$.

An example of a schedule of manufacturing processes in job-shop environment is presented in Fig. 1.

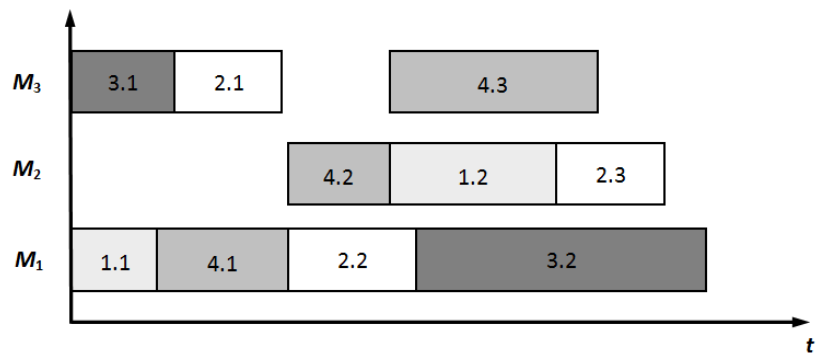

Figure 1. An example of a schedule in a job shop environment

\subsection{Limitations of job-shop system}

Literature in the field of job shop scheduling is mainly concerned with theoretical solutions $[6,10,11]$. This is a consequence of the solutions being still based on the classic set of simplifications [2]:

1. No two tasks of the same job can be scheduled in parallel.

2. Each machine is capable of carrying out one task at a time.

3. Each job has a limited number of operations of one per machine.

4. Each task is processed completely.

5. Task processing time can be entered manually.

6. Waiting time between two successive operations is allowed.

7. There are no two identical machines.

8. Machines can be idle. Machines perform one task at a time.

9. No machine failure. Machines are available throughout the whole manufacturing process.

10. All technological constraints are known and fixed. No variation is allowed.

11. No alternative process plans are allowed.

To a certain extent these assumptions are consistent with actual industrial conditions, however, several of these are against common practice. This concerns for instance assumption 9, "no machine failure," which is by no means a possible scenario in industrial conditions. Failure is inherent in the work of any machine, and practice shows that defects will eventually occur; that is why, an increasing amount of research work is done into diminishing theoretical constraints of job-shop problems. Literature shows several research trends, which seem to confirm those tendencies, e.g.:

job shop scheduling with alternative process plans - allowing for variation in technology constraint [12],

- flexible job-shop scheduling problem - concerned with job scheduling in flexible production systems [13],

- job shop scheduling with deteriorating jobs problem - accounting for the deterioration of working conditions over time [14],

- job shop scheduling under uncertainty - the trend which is focused on scheduling in an actual production environment; often referred to as robust scheduling $[15,16]$.

\subsection{Robust scheduling}

Robust scheduling of manufacturing jobs is a process taking into account variability of parameters of the production system, which produces a schedule characterised by its capability of absorbing disruptions $[17,18]$. The schedule is developed to counteract instability and nervousness connected with machine failure and randomness in production processes.
Robust scheduling is a combination of two methods for scheduling under uncertainty $[4,9]$ :

1. Predictive scheduling (off-line scheduling phase), belonging to the planning stage of the process, which is when the following schedules are developed:

- nominal schedule - based on actual parameters of a system

- robust schedule - taking into account uncertainty and flexibility of the executed process.

2. Reactive scheduling, which pertains to the execution stage and is referred to as on-line scheduling phase. The schedule is created or modified in production (any change of the process results in implementation of an alternative schedule).

Most common techniques of creating robust schedules include: redundancy-based techniques, conditional scheduling, creating partially ordered schedules, schedule sensitivity analysis [19].

In the next section we propose a new approach to predictive scheduling of manufacturing jobs with the implementation of selected prediction techniques. The suggested solution is designed to account for potential failure of the stock of machine tools by means of implementing redundant service times.

\section{Proposed machine failure prediction method}

\subsection{Survival analysis as a tool for machine failure prediction}

Prediction of selected uncertainty factors requires application of proper tools. The proposed solution makes use of elements of survival analysis, also referred to as survival time analysis.

Let $T$ be a non-negative random variable with probability density function $f(t), t>0$ and cumulative distribution function

$$
F(t)=P(T<t) .
$$

Bellow we assume, that the random variable $T$ represents the waiting time until the failure (death of plant). In literature the variable $T$ is called a survival time [21]. The value $F(t)$ determines the probability that the failure (defect) has occurred by duration. The survival function

$$
S(t)=P(T \geq t)=1-F(t)=\int_{t}^{\infty} f(s) d s
$$

presents the probability of correct work of device just before duration $t$ (the probability of surviving to duration $t$ ), generally the probability that the failure (defect) has not occurred by duration $t$. The survival characteristic of device may be presented by a hazard function

$$
h(t)=\lim _{d t \rightarrow 0} \frac{P(t \leq T<t+d t \mid T \geq t)}{d t}=\lim _{d t \rightarrow 0} \frac{\int_{t}^{t+d t} f(s) d s}{d t P(T \geq t)}=\frac{f(t)}{S(t)}
$$

The value of this function represents an instantaneous rate of occurrence of failure [19]. From (6) the formula (7) we may rewrite as

$$
h(t)=-\frac{d}{d t} \ln S(t)
$$

By solving the expression (8) we obtain a formula for survival function

$$
S(t)=\exp (-H(t))
$$

where $H(t)=\int_{0}^{t} h(s) d s$ is called a cumulative hazard function. The cumulative hazard function represents the sum of risks occurring from duration 0 to $t[20]$.

\subsection{Determination of time-to-failure}

To determine time-to-failure, our solution employs the survival function $S(t)$, derived from the Kaplan-Meier estimation, which is among techniques used in the field of survival analysis [20]. For the sake of analysis, the time-between-failures of machines was obtained from 
historical data, collected by the Maintenance Department of a production company. Such records are used inter alia in determining MTBF factor (Mean Time Between Failures). Statistical analysis of this data allows an engineer to determine frequently occurring time periods between machine failures. The resulting plot of horizontal steps may be used to estimate the chance of survival of a given machine (continuous survival time of machine) in defined periods (Fig. 2).

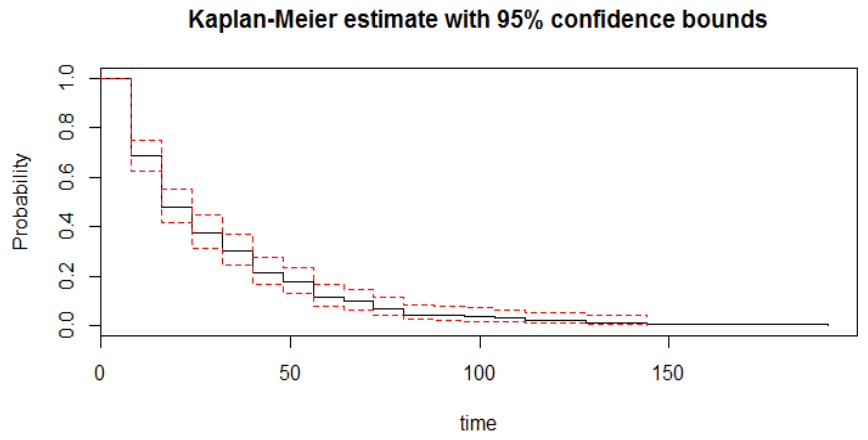

Figure 2. Survival function estimated with the Kaplan-Meier method

Time-to-failure prediction algorithm in the proposed solution consists of the following steps:

Step 1. Determining empirical time between failures.

Step 2. Determining the character and parameters of analysed data with the box plot (minimal and maximal values, scatter, upper and lower quantiles).

Step 3. Identifying periods of highest machine failure intensity

Step 4. Employing Kaplan-Meier estimator to determine the probability $p$ of uninterrupted processing

The process of data analysis presented above serves to pinpoint places where disruptions in the schedule could occur, whereas obtained probability $p$ will be applicable in implementing service times in the schedule.

\subsection{Estimating the size of service times}

It is critical for robust scheduling of jobs with redundant service times to determine their size. These may be derived from historical data, which indicate most frequent machine service times after failure. Fig. 3 shows an example of how a histogram may be used to determine the size of service times: first, the service times are extracted from the whole set of data, subsequently ensues a detailed analysis of machine repair times from the set of the highest number of machine failure incidences.
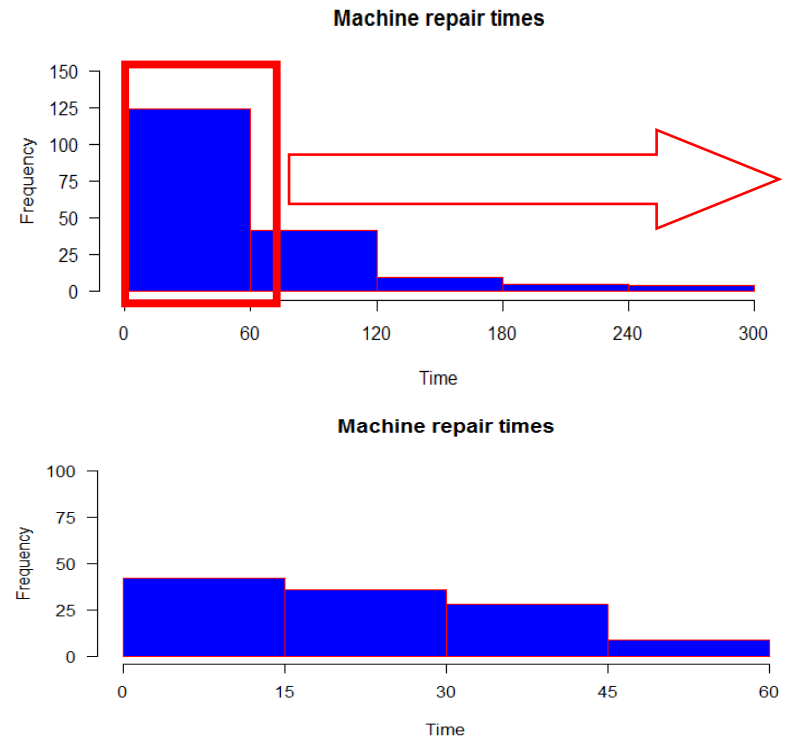

Figure 3. Historiograms of machine repair times

Given the potential failure time $t$ and probability $p$, the rule for the estimation of service times may take the following form:

IF $p<0.8$ and $p>0.6$ then minimal service buffer

ELSEIF $p<0.6$ and $p>0.4$

then medium service buffer

$$
\begin{aligned}
& \text { ELSEIF } p<0.4 \\
& \text { then maximal service buffer } \\
& \text { END }
\end{aligned}
$$

\section{Computational example}

\subsection{Model constraints}

In order to verify the proposed solution, a computational example was analysed. The constraints used in the model are presented below.

Production consists of 8 elements (jobs) of defined machine route in the stock of technological machines composed of 10 work stations in technological lay-out. Each element is a separate product, hence any job release and due dates are arbitrary. Elements are produced in batches of 75 pieces.

The production must be processed in the shortest possible time.

It was furthermore assumed that the manufacturing process may be subject to disruptions in the form of technological machine failure, which may occur on 5 machines of the heaviest workload. Failure prediction was based on analysis of historical data from a production company and calculated by means of the presented solution.

\subsection{Job scheduling method}

The selection of a suitable scheduling method is critical to scheduling manufacturing processes. The presented solution employs heuristic algorithm of dispatching rules. The method schedules jobs on particular machines (groups of machines) based on certain priority rules, according to which subsequent operations are chosen. There are several advantages of the method: quick solution, analysis of alternative scheduling scenarios or easy implementation of the method [22,23].

In the presented study, the following priority rules were employed:

1. LPT - Longest Processing Time,

2. SPT - Shortest Processing Time,

3. FCFS - First Come First Service,

4. EDD - Earliest Due Date,

5. RAND - Random.

\subsection{Criteria}

Both nominal and robust schedules must undergo evaluation. In the presented case the regular criteria for the evaluation of schedules are $[19,24,25]$ :

1. Make-span, total production time $\boldsymbol{C}_{\max }$ - the time when all the jobs end.

2. Job completion time $C_{i}$ and mean completion time of job $\boldsymbol{C}$ the time of completion of a given job $J_{i}$ and mean time of job completion, which is an arithmetic mean of all job completion times.

3. Extend - indicator based on the values of effectiveness criteria (e.g. $C_{\max }, C_{i}$ ) applied in reactive scheduling, although equally suitable in describing predictive schedule, for instance - to establish the maximum make-span of all jobs $C_{\max }$, we make use of the relationship:

$\frac{C_{\max }}{C_{\text {max }}^{\prime}}$

where: $\quad C_{\max }, C_{\max }^{\prime}-$ make-span of a nominal and robust schedule.

4. Number of critical operations - indicator used in evaluation of predictive schedules, defined as:

$\sum_{i} \sum_{j} y_{i j}$ 
$t r_{i+1}$ - release date of operation $i+1$ (subsequent).

An operation is therefore referred to as critical when there is no machine idle time between operations, as the risk of delay is higher.

5. Mean idle time between operations - obtained from: $\frac{\sum_{i} \sum_{j} t i_{i j}}{n_{t i}}$,

where: $\quad t i_{i j}-$ idle time preceding operation $j$ of job $i$, $n_{t i}-$ the number of idle times.

\subsection{Developing robust schedule}

The proposed solution was employed to conduct failure prediction of 5 technological machines. The results obtained from the analysis showed that machine failure occurs at $8,16,25$ or 32 hours of processing jobs. Given the processing of all jobs in the nominal schedule took on average approx. $27 \mathrm{~h}$, only times within the given period were considered. The survival functions determined for each machine gave the probability of uninterrupted processing $p$. Based on the obtained data and the machine defect repair times the service times $t s_{i}$ were established. The results of analyses are collated in Table 1.

Table 1. Service times of analysed machines

\begin{tabular}{|c|c|c|c|c|c|c|c|c|c|c|}
\hline & \multicolumn{2}{|c|}{ Machine 1} & \multicolumn{2}{|c|}{ Machine 2} & \multicolumn{2}{|c|}{ Machine 3} & \multicolumn{2}{|c|}{ Machine 4} & \multicolumn{2}{|c|}{ Machine 5} \\
\hline & $p$ & $\begin{array}{l}t s_{1} \\
{[\mathrm{~h}]}\end{array}$ & $p$ & $\begin{array}{l}t S_{2} \\
{[\mathrm{~h}}\end{array}$ & $p$ & \begin{tabular}{|l}
$t s_{3}$ \\
{$[\mathrm{~h}]$}
\end{tabular} & $p$ & \begin{tabular}{|l}
$t s_{4}$ \\
{$[\mathrm{~h}$}
\end{tabular} & $p$ & \begin{tabular}{|l|l}
$t S_{5}$ \\
{$[\mathrm{~h}$}
\end{tabular} \\
\hline After $8 \mathrm{~h}$ & 0.757 & 0.25 & \begin{tabular}{|l}
0.715 \\
\end{tabular} & 0.25 & 0.754 & \begin{tabular}{|l|l} 
\\
\end{tabular} & 0.592 & 0.5 & 0.684 & 0.25 \\
\hline After $16 \mathrm{~h}$ & 0.549 & \begin{tabular}{|l|}
0.5 \\
\end{tabular} & 0.515 & \begin{tabular}{|l|}
0.5 \\
\end{tabular} & 0.566 & 0.5 & \begin{tabular}{|l|}
0.401 \\
\end{tabular} & 0.5 & 0.481 & \begin{tabular}{|l|}
0.5 \\
\end{tabular} \\
\hline
\end{tabular}

The obtained data provided information necessary for building a robust schedule by implementing service times where machine failure might occur. Buffer times were scheduled on machines $M_{1}, M_{4}, M_{5}, M_{9}$ and $M_{10}$, and the scheduling algorithm took the following form:

IF there is a free spot in nominal schedule, put service time $t s_{i}$

ELSEIF operation is scheduled in service time target spot in nominal schedule check condition:

IF inserting time service does not affect objective function schedule ts in target spot and move operations to the right

ELSE

schedule service time ts after completed operation END

END

\subsection{Results and discussion}

By implementing the above instructions we obtain the nominal and the robust schedule.

The objective functions for both schedules are presented in Table 2, where it can be seen that different dispatching rules produced different values of objective function and parameters in question.

LPT dispatching rule produced the longest make-span. In the case of FCFS and EDD the objective function was average, whereas the shortest makespan resulted from the implementation of SPT rule, which led us to a conclusion that the robust schedule should not include service times after $16 \mathrm{~h}$ of the preceding failure. Similarly positive results were obtained when RAND rule was employed, however, it is difficult to predict what the result would be if the random algorithm were to be run again.

The comparison of objective functions in the nominal and the robust schedules indicates that prediction of machine failure and scheduling service times does not overburden the $C_{\max }$ parameter (longer by a maximum of $0.75 \mathrm{~h}$ ), and in the case of one method the objective function value is even better (the make-span is shortened by $0.6 \mathrm{~h}$ ). This can also be observed in the relation of $C_{\max }$ and $C_{\max }^{\prime}$, which takes the value of 1 in each analysed case, hence the schedules are broadly similar to each other.

Table 2. Obtained values of objective function $C_{\max }$

\begin{tabular}{|c|c|c|c|c|}
\hline $\begin{array}{l}\text { Dispatching } \\
\text { rules }\end{array}$ & $\begin{array}{l}\text { Nominal } \\
\text { schedule } \\
C_{\max }[\mathrm{h}]\end{array}$ & $\begin{array}{l}\text { Robust } \\
\text { schedule } \\
C_{\max }^{\prime}[\mathrm{h}]\end{array}$ & $\begin{array}{l}\text { Extend } \\
\frac{C_{\max }}{C_{\max }^{\prime}}\end{array}$ & $\begin{array}{l}\text { Difference } \\
C_{\max }-C_{\max }^{\prime}[\mathbf{h}]\end{array}$ \\
\hline LPT & 30.88 & 31.38 & 0.97 & 0.5 \\
\hline SPT & 22.09 & 22.84 & 0.97 & 0.75 \\
\hline FCFS & 25.78 & 26.53 & 0.97 & 0.75 \\
\hline EDD & 25.78 & 26.53 & 0.97 & 0.75 \\
\hline RAND & 25.45 & 24.85 & 1.02 & -0.6 \\
\hline
\end{tabular}

Since the $C_{\max }$ obtained from EDD rule was on average level among other rules, it was resolved that the remaining parameters (Table 3 , Table 4 and Table 5) will be established from the same rule.

It should be noted that, on average, due times of particular jobs in the robust schedule are longer by $0.83 \mathrm{~h}$, however, this improves the robustness of the schedule by ensuring that no loss of performance occurs in the presence of disruption - in 5 out of 8 processed jobs the number of critical operations was reduced (Table 4). What is more, analysis of idle times (Table 5) proves that in the majority of cases service times were implemented in machine idle times, therefore utilising the time when the machine does not process any jobs. It was only in the case of $M_{3}$ that the make-span was bigger.

Table 3. Obtained due dates of jobs

\begin{tabular}{|l|l|l|l|l|l|l|l|l|l|}
\cline { 2 - 9 } \multicolumn{1}{c|}{} & \multicolumn{1}{l|}{ Value of $\boldsymbol{C}_{\boldsymbol{i}}$} \\
\cline { 2 - 9 } & $C_{1}$ & $C_{2}$ & $C_{3}$ & $C_{4}$ & $C_{5}$ & $C_{6}$ & $C_{7}$ & $C_{8}$ & $\bar{C}$ \\
\hline Nominal & 3.7 & 20.3 & 10.5 & 14.5 & 21.3 & 19.3 & 25.7 & 13.4 & $\mathbf{1 6 . 1}$ \\
schedule & 1 & 3 & 5 & 7 & 8 & 3 & 8 & 5 & $\mathbf{4}$ \\
\hline $\begin{array}{l}\text { Robust } \\
\text { schedule }\end{array}$ & 3.7 & 21.3 & 12.3 & 15.3 & 22.4 & 20.3 & 26.0 & 14.2 & $\mathbf{1 6 . 9}$ \\
\hline
\end{tabular}

Table 4. The number of critical operations in nominal and robust schedule

\begin{tabular}{|l|l|l|l|l|l|l|l|l|l|}
\cline { 2 - 11 } \multicolumn{1}{c|}{} & \multicolumn{7}{|c|}{ Number of critical operations } \\
\cline { 2 - 11 } & $J_{1}$ & $J_{2}$ & $J_{3}$ & $J_{4}$ & $J_{5}$ & $J_{6}$ & $J_{7}$ & $J_{8}$ & $\bar{J}$ \\
\hline Nominal schedule & 1 & 2 & 2 & 3 & 2 & 4 & 4 & 5 & $\mathbf{3}$ \\
\hline Robust schedule & 1 & 1 & 2 & 1 & 2 & 3 & 3 & 4 & $\mathbf{2}$ \\
\hline
\end{tabular}

Table 5. Average idle time of machines

\begin{tabular}{|l|l|l|l|l|l|l|}
\cline { 2 - 7 } \multicolumn{1}{c|}{} & \multicolumn{6}{|c|}{ Average idle time of machines } \\
\cline { 2 - 7 } & $M_{1}$ & $M_{2}$ & $M_{3}$ & $M_{4}$ & $M_{5}$ & $\bar{M}$ \\
\hline Nominal schedule & 0.00 & 3.15 & 3.66 & 2.52 & 4.04 & $\mathbf{2 . 6 7}$ \\
\hline Robust schedule & 0.00 & 3.15 & 6.00 & 2.20 & 4.25 & $\mathbf{3 . 1 2}$ \\
\hline
\end{tabular}

The schedule developed in the form of Gantt chart is show in Fig. 4. Service times are red fields with bold frame.

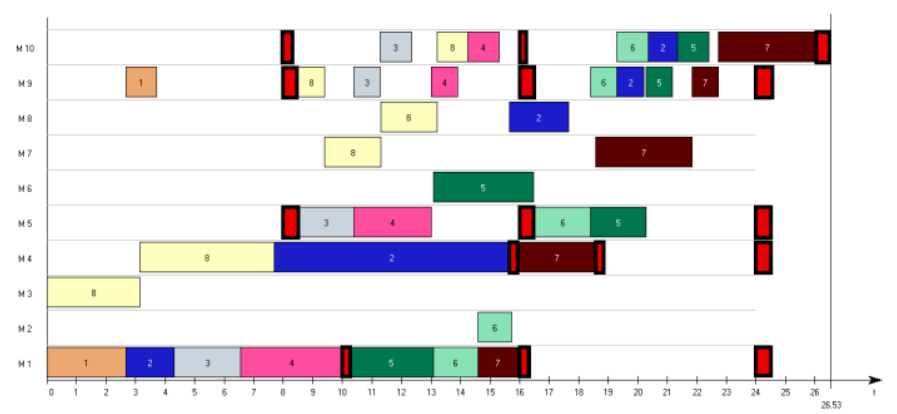

Figure 4. Developed manufacturing process schedule

\section{Summary}

Scheduling jobs in manufacturing processes is of great assistance to production management.

The available methods and techniques must, however, be developed in order to embrace an increasing number of factors, and to give a more actual representation of existing production systems. 
The presented research work aimed at showing that the development of a robust schedule with the implementation of service times is feasible given that slight adjustments to the manufacturing process schedule parameters are introduced. Future work should concentrate on optimisation of the developed nominal schedule so that it would bear high resemblance to the robust schedule. It is furthermore recommended that simulation research should be carried out with a view to further validation of the proposed solution.

\section{References}

[1] Kłosowski G., Gola A., Świć A., Application of Fuzzy Logic in Assigning Workers to Production Tasks Advances in Intelligent Systems and Computing, Vol. 474, 2016, pp. 505-513.

[2] S. French: Sequencing and Scheduling: An Introduction to the Mathematics of the Job-Shop (J. Wiley \& Sons, New York, 1982).

[3] M. Klimek, P. Lebkowski: Resource allocation for robust project scheduling. Bulletion Of The Polish Academy Of Sciences-Technical Sciences, Vol. 59, Issue 1, pp. 51-55.

[4] Kłosowski G., Gola A., Risk-based estimation of manufacturing order costs with artificial intelligence [in:] Ganzha M., Maciaszek L., Paprzycki M. (eds.), Proceedings of the 2016 Federated Conference on Computer Science and Information Systems (FEDCSIS), IEEE, 2016, pp. 729-732.

[5] H. Bräsel, L. Dornheim, S. Kutz, M. Mörig, I. Rössling: LiSA - A Library of Scheduling Algorithms (Magdeburg University, 2001).

[6] Ł. Sobaszek, A. Gola: Computer-aided production task scheduling. Applied Computer Science, Vol. 11, No. 4, pp. 58-69.

[7] G. Vilcot, J-Ch. Billaut: A Tabu Search and a Genetic Algorithm for Solving a Bicriteria General Job Shop Scheduling Problem. European Journal of Operational Research, 190 (2008), pp. 398-411.

[8] A. K. Kaban, Z. Othman, D. S. Rohmah: Comparison of Dispatching Rules in Job-Shop Scheduling problem Using Simulation: A Case Study. International Journal of Simulation Modelling, Vol. 11, No. 3, 2012, pp. 129-140.

[9] Hong Gao: Bulding Robust Schedules using Temporal Potection - An Emipirical Study of Constraint Based Scheduling Under Machine Failure Uncertainty (Toronto, Ontario, 1996).

[10] H. E. Nouri, O. B. Driss, K. Ghedira: Hybrid Metaheuristics within a Holonic Multiagent Model for the Flexible Job Shop Problem. Procedia Computer Science, 60, (2015), pp. 83-92.

[11] Ch. S. Thomalla: Job shop scheduling with alternative process plans, Int. J. Production Economics, 74 (2001), pp. 125-134.
[12] In-Chan Choi, Dae-Sik Choi: A Local Search Algorithm for Jobshop Scheduling Problems with Alternative Operations and SequenceDependent Setups. Computers \& Industrial Engineering, 42 (2002), pp. 43-58.

[13] Kai Zhou Gao, Ponnuthurai Nagaratnam Suganthan, Tay Jin Chua, Chin Soon Chong, Tian Xiang Cai, Qan Ke Pan: A Two-Stage Artificial Bee Colony Algorithm Scheduling Flexible Job-Shop Scheduling Problem with New Job Insertion. Expert Systems with Applications, Vol. 42, Issue 21, 2015, pp. 7652-7663.

[14] Ji-Bo Wang, Lu Liu, Cheng Wang: Single Machine SLK/DIF Due Window Assignment Problem with Learning Effect and Deteriorating Jobs. Applied Mathematical Modelling, Vol. 37, Issues 18-19, 2013, pp. 83948400.

[15] V. J. Leon, S. D. Wu, R. H. Storer: Robustness Measures and Robust Scheduling for Job Shops. IIE transactions, 26(5), 1994, pp. 32-43.

[16] Nasr Al-Hinai, T. Y. ElMekkawy: Robust and Stable Flexible Job Shop Scheduling with Random Machine Breakdowns Using a Hybrid Genetic Algorithm. International Journal of Production Economics, Vol. 132, Issue 2, 2011, pp. 279-291.

[17] S. Kłos, J. Patalas-Maliszewska, P. Trebuna: Improving Manufacturing Processes Using Simulation Methods. Applied Computer Science, Vol. 12, No, 4, 2016, pp. 7-17.

[18] R. Knosala: Zastosowania metod sztucznej inteligencji w inżynierii produkcji (Wydawnictwa Naukowo-Techniczne, Warszawa 2002).

[19] G. E. Vieira, J. W. Herrmann, E. Lin: Rescheduling Manufacturing Systems: A Framework of Strategies, Policies, and Methods. Journal of Scheduling, 6, 2003, pp. 39-62.

[20] D. W. Hosmer, Jr., S. Lemeshow, S. May: Applied survival analysis: regression modeling of time to event data (2nd edition) (John Wiley \& Sons, 2008).

[21] J. F. Lawless, Statistical models and methods for lifetime data (John Wiley \& Sons, 2003).

[22] T. C. Chiang, L. C. Fu: Using dispatching rules for job shop scheduling with due date-based objectives. International Journal of Production Research, vol. 45(14), May 2007, pp. 1-28.

[23] Mujanah Ezat Agha, Simulation of Production Scheduling in Manufacturing Systems (Dublin City, 1993).

[24] Deepu P., Robust Schedules and Disruption Management for Job Shops (Bozeman, Montana, 2008).

[25] Bibo Yang, J. Geunes: Predictive-reactive scheduling on a single resource with Uncertain Future Jobs. European Journal of Operational Research, Vol. 189, Issue 3, 2008, pp. 1267-1283. 